\title{
Conceptual K-Means Algorithm Based on Complex Features
}

\author{
I.O. Ayaquica-Martínez, J.Fco. Martínez-Trinidad, and J. Ariel Carrasco-Ochoa
}

\author{
National Institute of Astrophysics, Optics and Electronics \\ Computer Science Department \\ Luis Enrique Erro \# 1, Santa María Tonantzintla, Puebla, Mexico, C.P. 72840 \\ \{ayaquica, fmartine, ariel\}@inaoep.mx
}

\begin{abstract}
The k-means algorithm is the most studied and used tool for solving the clustering problem when the number of clusters is known a priori. Nowadays, there is only one conceptual version of this algorithm, the conceptual k-means algorithm. One characteristic of this algorithm is the use of generalization lattices, which define relationships among the feature values. However, for many applications, it is difficult to determine the best generalization lattices; moreover there are not automatic methods to build the lattices, thus this task must be done by the specialist of the area in which we want to solve the problem. In addition, this algorithm does not work with missing data. For these reasons, in this paper, a new conceptual k-means algorithm that does not use generalization lattices to build the concepts and allows working with missing data is proposed. We use complex features for generating the concepts. The complex features are subsets of features with associated values that characterize objects of a cluster and at the same time not characterize objects from other clusters. Some experimental results obtained by our algorithm are shown and they are compared against the results obtained by the conceptual k-means algorithm.
\end{abstract}

\section{Introduction}

The conceptual clustering problem was first addressed in the 80's by Michalski [1]. It consists on finding, from a data set, not only the clusters but also a conceptual interpretation of them. Starting from the Michalski's works several algorithms have been developed to solve the conceptual clustering problem. Some of them can be found in [2-8].

The k-means algorithm is the most studied and used tool for solving the clustering problem when the number of clusters is known a priori. The conceptual k-means algorithm proposed by Ralambondrainy [8] is the unique conceptual version of this algorithm. Then, we are going to focus in this algorithm.

The conceptual k-means algorithm [8] was developed to solve problems where the number of clusters is known a priori. This algorithm consists of two phases: an aggregation phase, in which the clusters are built and a characterization phase, in which the concepts are generated. In the aggregation phase, the k-means algorithm was extended to work with mixed data. In order to solve the mixed data problem, a 
distance function given as the weighted sum of the Euclidean distance, for quantitative features; and the Chi-square distance, for qualitative features was used. For applying the Chi-square distance, a transformation of each qualitative feature into a set of Boolean features, must be done. The new features are handled as numeric features, which is inadequate. Additionally, this distance function does not work with missing data. On the other hand, this algorithm uses the same distance function for comparing objects in any problem.

In the characterization phase, a generalization lattice for each feature is required. For the qualitative features, it is difficult to determine the best generalization lattice for each feature; moreover there are not automatic methods to build the lattices, therefore this task must be done by the specialist; while for the quantitative features a codification into qualitative features to allow building a generalization lattice is done. In addition, the missing data is not taken into account when the generalization lattice is built.

In this paper, we propose a new conceptual k-means algorithm, which allows working with mixed and missing data; our algorithm does not require any transformation of features neither generalization lattices for the construction of the concepts.

This paper is organized as follows: in Section 2, some basic concepts are given. In Section 3, we introduce a new conceptual clustering algorithm. In Section 4, a measure to evaluate the quality of the concepts is proposed. The tests made with the proposed algorithm are shown in Section 5. In Section 6, a comparison between the conceptual k-means algorithm and the proposed algorithm is presented. Finally, in Section 7, conclusions and future work are presented.

\section{Basic Concepts}

The goal of this paper is to propose a new conceptual clustering algorithm, such that similar objects belong to the same cluster while dissimilar objects belong to different clusters. Besides, the concept associated to each cluster should characterize objects of the cluster and at the same time not characterize objects from other clusters. The complex features [9] are combinations of values for a subset of features such that these values appear sufficiently in the objects into a cluster and at the same time not appear in the objects outside the cluster. For this reason, they could be used to generate concepts.

The formal outline of the problem is the following:

Let $U=\left\{O_{1}, \ldots, O_{m}\right\}$ be a set of $m$ objects. Each object is described by a set of $n$ features $R=\left\{x_{1}, \ldots, x_{n}\right\}$. Each feature $x_{i}$ takes values in a set of admissible values, $D_{i}$, $i=1, \ldots, n$. The features can be of any nature (qualitative: Boolean, multi-valued, etc. or quantitative: integer, real).

Also, it is assumed that in $D_{i}$ exists a symbol "?" that denotes missing data, so incomplete descriptions of objects can be considered.

For each feature $x_{i}$, a comparison function $F C_{i}: D_{i} \times D_{i} \rightarrow L_{i}, i=1, \ldots, n$ is defined, where $L_{i}$ is a completely ordered set. The $F C_{i}$ function is an evaluation of the similarity degree between two values of the feature $x_{i}$. In addition, let 
$\Gamma:\left(D_{1} \times \ldots \times D_{n}\right)^{2} \rightarrow[0,1]$ be a similarity function, which allows evaluating the similarity degree between two object descriptions.

The conceptual clustering problem consists on structuring the objects in $k$ clusters and also generating the concept associated to each cluster.

As we mention above, the complex features could be used for generating concepts because, they are subsets of features with associated values that characterize objects of a cluster and at the same time not characterize objects from other clusters.

In order to obtain the complex features, some subsets of features, $\Omega$, denominated support sets are needed. The support sets indicate the parts of the objects where the complex features will be searched. These support sets can be obtained in different ways. In this paper, the $\Gamma$-discriminating, the $\Gamma$-characterizing and support sets that are $\Gamma$-discriminating and $\Gamma$-characterizing at the same time are used.

In the following definitions, $\Omega O$ is a subdescription of the object $O$ taking into account only the features of $\Omega$.

1) $\Gamma$-discriminating support sets are subsets of features that do not increase the similarity among the objects from different clusters and they are defined as follows:

Def. 1: $\Omega \subseteq R$ is a $\Gamma$-discriminating support set of features if all pairs of objects $\left(O_{i}, O_{j}\right)$ of different clusters satisfy $\Gamma\left(\Omega O_{i}, \Omega O_{j}\right) \leq \Gamma\left(O_{i}, O_{j}\right)$, i.e., $\Omega$ is a $\Gamma$ discriminating support set of features, if objects in different clusters do not have a greater similarity in $\Omega$ than in $R$.

2) $\Gamma$-characterizing support sets are subsets of features that do not reduce the similarity among the objects in the same cluster and they are defined as follows:

Def. 2: $\Omega \subseteq R$ is a $\Gamma$-characterizing support set of features if all pairs of objects $\left(O_{i}, O_{j}\right)$ in the same cluster satisfy $\Gamma\left(O_{i}, O_{j}\right) \leq \Gamma\left(\Omega O_{i}, \Omega O_{j}\right)$, i.e., $\Omega$ is a $\Gamma$ characterizing support set of features, if objects of the same cluster do not have a lesser similarity in $\Omega$ than in $R$.

3) Support sets that are $\Gamma$-discriminating and $\Gamma$-characterizing at the same time.

For calculating the support sets, the degree in which each subset $\Omega$ satisfies the $\Gamma$ discriminating, $\Gamma$-characterizing or both properties, respectively is evaluated. It is to say:

1) For $\Gamma$-discriminating support sets, the degree in which the subset $\Omega$ satisfies the definition of $\Gamma$-discriminating support set is evaluated measuring the number of pairs of objects in different clusters, such that their similarity in $\Omega$ is lesser or equal than their similarity in $R$, as well as how much their similarity values differ in $\Omega$ and $R$.

2) For $\Gamma$-characterizing support sets, the degree in which the subset $\Omega$ satisfies the definition of $\Gamma$-characterizing support set is evaluated measuring the number of pairs of objects in the same cluster, such that their similarity in $\Omega$ is greater or equal than their similarity in $R$, as well as how much their similarity values differ in $R$ and $\Omega$.

3) For support sets that are $\Gamma$-discriminating and $\Gamma$-characterizing at the same time, the degree in which the subset $\Omega$ satisfies both definitions is evaluated. This degree 
is evaluated as the sum of the degree in which the subset $\Omega$ satisfies the definition of $\Gamma$-discriminating support set and the degree in which the subset $\Omega$ satisfies the definition of $\Gamma$-characterizing support set.

Additionally to the support sets it is also necessary a set of values associated to these features, such that these values appear sufficiently in the objects into the cluster and at the same time do not appear in the objects outside the cluster. For this reason we use the complex features.

Def. 3: Let $\Omega=\left\{x_{i_{1}}, \ldots, x_{i_{p}}\right\}$ be a support set and $\left(a_{1}, \ldots, a_{p}\right)$ values associated to the features $x_{i_{1}}, \ldots, x_{i_{p}}$ from an object of the sample, then $\left\{x_{i_{1}}, \ldots, x_{i_{p}}\right\}$ and $\left(a_{1}, \ldots, a_{p}\right)$ form a complex feature of the cluster $A_{i}$, if and only if:
1) $\sum_{O \in A_{i}} \Gamma\left(\Omega O,\left(a_{1}, \ldots, a_{p}\right)\right) \geq \beta_{i}$
2) $\sum_{O \notin A_{i}} \Gamma\left(\Omega O,\left(a_{1}, \ldots, a_{p}\right)\right)<\lambda_{i}$

where $\beta_{i}$ is the minimum similarity that the objects of the cluster $A_{i}$ should have with the subdescription $\left(a_{1}, \ldots, a_{p}\right)$ and $\lambda_{i}$ is the maximum similarity that the objects outside of the cluster should have with $\left(a_{1}, \ldots, a_{p}\right)$. For this work, we used $\lambda_{i}=1$, because we are interested in obtaining concepts, with the smallest number of complex features, that do not recognize objects of other clusters. We tried $\lambda_{i}=0$ but there were some clusters without any complex feature. Also, we tried $\lambda_{i}=2$ and in this case we obtained too much complex features. The value of $\beta_{i}$ is calculated automatically. The procedure to calculate $\beta_{i}$ is the following: Initially, $\beta_{i}$ is the number of objects in the cluster; then $\beta_{i}$ is decremented taking as value the maximum similarity obtained by the subdescriptions that do not satisfy the complex feature definition. Then the complex features are computed with the new value of $\beta_{i}$; this process is repeated while $\beta_{i}>0$.

\section{Conceptual K-Means Algorithm Based on Complex Features}

We will follow the same idea of Ralambondrainy proposing a conceptual k-means algorithm in two phases: a clustering phase, in which the k-means with similarity functions algorithm (KMSF) [10] is used to obtain the clusters; and a characterization phase, in which the complex features are used to generate the concepts.

\subsection{Clustering Phase}

In this phase, the k-means with similarity functions algorithm is used to build the clusters. This algorithm allows working with mixed, qualitative and quantitative, features defining a similarity function. In this way, the similarity function does not require transformations of features as the distance of the conceptual k-means algorithm does. Also, the similarity function is usually defined in terms of comparison functions, which express how the values of the features are compared. This algorithm 
contrary to the conceptual k-means algorithm (CKM) allows using different functions to compare the values of features depending on the problem to solve.

For example, in this work we use the following similarity function:

$$
\Gamma\left(O_{i}, O_{j}\right)=\frac{\sum_{x_{s} \in R} F C_{s}\left(x_{s}\left(O_{i}\right), x_{s}\left(O_{j}\right)\right)}{|R|}
$$

where $F C_{s}\left(x_{s}\left(O_{i}\right), x_{s}\left(O_{j}\right)\right)$ is the comparison function used for comparing two values of the feature $x_{s}$.

The comparison functions used for the tests were the following:

i) For quantitative features:

$$
F C_{s}\left(x_{s}\left(O_{i}\right), x_{s}\left(O_{j}\right)\right)= \begin{cases}0 & \text { if } x_{s}\left(O_{i}\right)=? \vee x_{s}\left(O_{j}\right)=? \vee\left|x_{s}\left(O_{i}\right)-x_{s}\left(O_{j}\right)\right| \geq \sigma \\ 1 & \text { in other case }\end{cases}
$$

where $\sigma$ is the standard deviation for the feature $x_{s}$.

ii) For qualitative features:

$$
F C_{s}\left(x_{s}\left(O_{i}\right), x_{s}\left(O_{j}\right)\right)= \begin{cases}0 & \text { if } x_{s}\left(O_{i}\right)=? \vee x_{s}\left(O_{j}\right)=? \vee x_{s}\left(O_{i}\right) \neq x_{s}\left(O_{j}\right) \\ 1 & \text { in other case }\end{cases}
$$

Notice that, when a value for the feature is missing then the value is different from any other value even from other missing value.

On the other hand, the KMSF algorithm, selects objects of the sample as centroids of the clusters (see details in [10]) instead of the mean, as occur in the modified kmeans algorithm proposed by Ralambondrainy [8].

\subsection{Characterization Phase}

In this phase, the complex features are used to generate the concepts. In order to use the complex features, some support sets are needed. In this work, the $\Gamma$-discriminating $(\Gamma \mathrm{d}), \Gamma$-characterizing $(\Gamma \mathrm{c})$ and $\Gamma$-discriminating and $\Gamma$-characterizing $(\Gamma \mathrm{d}$ and $\Gamma \mathrm{c})$ support sets are used.

For calculating the support sets, a genetic algorithm was used. In this genetic algorithm, each individual represents a support set and it is formed by $n$ genes, where each gene represents a feature. A gene takes value 1 if the feature is included and 0 if it is not included. This algorithm uses the 1-point crossover operator, it is to say, a cross point is selected and starting from it, the information of the chromosomes is exchanged. As mutation operator, the uniform mutation is used, it is to say, a gene of a chromosome is randomly selected and its value is changed ( 0 by 1 or 1 by 0 ). As aptitude function, the degree in which a subset $\Omega$ satisfies the definition of $\Gamma$ discriminating, $\Gamma$-characterizing or both respectively is used. From these sets, those that satisfy with the highest degree the $\Gamma$-discriminating property, $\Gamma$-characterizing property or both, respectively, are selected.

Example: For the sample of Table 1, suppose that after applying the clustering phase, the following clusters were obtained: $A_{1}=\left\{O_{1}, O_{2}\right\}$ and $A_{2}=\left\{O_{3}, O_{4}\right\}$.

For these clusters, the genetic algorithm obtained the support sets shown in Table 2. 
Table 1. Sample with four objects described by three features

\begin{tabular}{|c|c|c|c|}
\hline \multirow{2}{*}{ Objects } & \multicolumn{3}{|c|}{ Features } \\
\cline { 2 - 4 } & Color $(\mathbf{C})$ & Weight $(\mathbf{W})$ & Shape (Sh) \\
\hline $\mathrm{O}_{1}$ & Red & 20 & Round \\
\hline $\mathrm{O}_{2}$ & Red & 25 & Round \\
\hline $\mathrm{O}_{3}$ & Yellow & 30 & Triangle \\
\hline $\mathrm{O}_{4}$ & Yellow & 35 & Triangle \\
\hline
\end{tabular}

Table 2. Support sets obtained by the genetic algorithm for the sample of the Table 1 with $A_{1}=\left\{O_{1}, O_{2}\right\}$ and $A_{2}=\left\{O_{3}, O_{4}\right\}$

\begin{tabular}{|c|c|c|}
\hline \multicolumn{3}{|c|}{ Support sets } \\
\hline$\Gamma \mathbf{d}$ & $\Gamma \mathbf{c}$ & $\Gamma \mathbf{d} \mathbf{y} \boldsymbol{\Gamma}$ \\
\hline$\{\mathrm{C}\}$ & \multirow{2}{*}{$\mathrm{C}, \mathrm{Sh}$} & $\{\mathrm{C}, \mathrm{W}, \mathrm{Sh}\}$ \\
\hline$\{\mathrm{C}, \mathrm{W}\}$ & & $\{\mathrm{C}, \mathrm{W}\}$ \\
& & $\{\mathrm{W}\}$ \\
\hline
\end{tabular}

Once obtained the support sets, the complex features are obtained applying the definition 3.

In this phase, the similarity function used to calculate the complex features is the same one used in the clustering phase. An advantage of using the same similarity function in both phases of the algorithm is that the way in which the concepts are generated maintains a relationship with the cluster construction. This does not happen in the conceptual k-means algorithm, where the comparison used to generate the concepts is different from the one used to build the clusters.

The complex features obtained with the support sets of Table 2 and the dataset of Table 1 are shown in Table 3.

Table 3. Complex features for the example

\begin{tabular}{|c|c|c|c|}
\hline & \multicolumn{3}{|c|}{ Complex Features for each support set } \\
\hline Cluster & $\Gamma \mathrm{d}$ & $\Gamma \mathrm{c}$ & $\Gamma \mathrm{d} \mathbf{y} \Gamma \mathrm{c}$ \\
\hline 1 & $\begin{array}{c}\{\mathrm{C}\}-(\text { Red }) \\
\{\mathrm{C}, \mathrm{W}\}-(\text { Red }, 20) \\
\{\mathrm{C}, \mathrm{W}\}-(\text { Red,25) } \\
\{\mathrm{W}\}-(20)\end{array}$ & $\{\mathrm{C}, \mathrm{Sh}\}-($ Red,Round $)$ & $\begin{array}{c}\{\mathrm{C}, \mathrm{W}, \mathrm{Sh}\}-(\text { Red,20,Round }) \\
\{\mathrm{C}, \mathrm{W}, \mathrm{Sh}\}-(\text { Red,25,Round }) \\
\{\mathrm{C}, \mathrm{W}\}-(\text { Red }, 20) \\
\{\mathrm{C}, \mathrm{W}\}-(\text { Red,25) } \\
\{\mathrm{W}\}-(20)\end{array}$ \\
\hline 2 & $\begin{array}{c}\{\mathrm{C}\}-(\text { Yellow }) \\
\{\mathrm{C}, \mathrm{W}\}-(\text { Yellow,30) } \\
\{\mathrm{C}, \mathrm{W}\}-(\text { Yellow,35) } \\
\{\mathrm{W}\}-(35)\end{array}$ & $\{\mathrm{C}, \mathrm{Sh}\}-($ Yellow,Triangle $)$ & $\begin{array}{c}\mathrm{C}, \mathrm{W}, \mathrm{Sh}\}-(\text { Yellow,30,Triangle }) \\
\{\mathrm{C}, \mathrm{W}, \text { Sh }\}- \\
\text { (Yellow,35,Triangle }) \\
\{\mathrm{C}, \mathrm{W}\}-(\text { Yellow,30) } \\
\{\mathrm{C}, \mathrm{W}\}-(\text { Yellow,35) } \\
\{\mathrm{W}\}-(35)\end{array}$ \\
\hline
\end{tabular}

Afterwards, in order to generate the concepts, a predicate is associated to each complex feature. This predicate is built in the following way: to each feature $x_{i} \in R$ that appears in the complex feature, the associated value $a_{i}$ is assigned to this feature; and for the features $x_{i} \in R$ that do not appear in the complex feature $*$ is assigned. The symbol * means "any value is possible". 
For the example, the predicates formed for the complex features of $\Gamma$ discriminating $(\Gamma \mathrm{d})$ sets of the cluster 1 , in Table 3 are:

$$
\begin{aligned}
& \mathrm{P} 1: \mathrm{C}=\operatorname{Red} \wedge \mathrm{W}=* \wedge \mathrm{Sh}=* \\
& \mathrm{P} 2: \mathrm{C}=\operatorname{Red} \wedge \mathrm{W}=20 \wedge \mathrm{Sh}=* \\
& \mathrm{P} 3: \mathrm{C}=\operatorname{Red} \wedge \mathrm{W}=25 \wedge \mathrm{Sh}=* \\
& \mathrm{P} 4: \mathrm{C}=* \wedge \mathrm{W}=20 \wedge \mathrm{Sh}=*
\end{aligned}
$$

The set of predicates obtained from the complex features can contain two or more predicates that recognize the same objects. Therefore, this set of predicates can be reduced by eliminating predicates that recognize the same objects than another predicate. This reduction is made using the strategy proposed by Ralambondrainy [8] that works as follows: first, the predicates are sorted, in a descending way, according to the amount of objects that each one recognizes. The first predicate will be part of the concept. For the remaining predicates, if a predicate recognizes some objects not recognized by the stored predicates, then it is added to the concept; otherwise it is eliminated. The concept is formed by the disjunction of the stored predicates.

For the example, the concepts obtained after the reduction process are shown in Table 4.

Table 4. Concepts obtained for the example

\begin{tabular}{|c|c|c|}
\hline Kind of support set & Cluster & Concept \\
\hline \multirow{2}{*}{$\Gamma \mathrm{d}$} & 1 & $\mathrm{C}=$ Red \\
& 2 & $\mathrm{C}=$ Yellow \\
\hline \multirow{2}{*}{$\Gamma \mathrm{c}$} & 1 & $\mathrm{C}=$ Red $\wedge \mathrm{Sh}=$ Round \\
& 2 & $\mathrm{C}=$ Yellow $\wedge \mathrm{Sh}=$ Triangle \\
\hline \multirow{2}{*}{$\Gamma \mathrm{d} \Gamma \mathrm{c}$} & 1 & $\mathrm{C}=$ Red $\wedge \mathrm{W}=20 \wedge \mathrm{Sh}=$ Round \\
& 2 & $\mathrm{C}=$ Yellow $\wedge \mathrm{W}=30 \wedge \mathrm{Sh}=$ Triangle \\
\hline
\end{tabular}

\section{Quality Measure}

In 1995, Ralambondrainy proposed to take as a quality measure, the percentage of objects in the cluster that are recognized by the concept. However, we consider that it is also necessary to take into account the objects outside of the cluster that are recognized by the concept. Therefore, we propose the following quality measure:

$$
\text { quality }\left(C_{1}, \ldots, C_{k}\right)=\frac{1}{k} \sum_{i=1}^{k} \frac{\text { examples }\left(C_{i}\right)}{\text { total }\left(C_{i}\right)+\text { counterexamples }\left(C_{i}\right)}
$$

where:

\footnotetext{
$k \quad$ is the number of clusters.

$C_{i}$ is the concept associated to the $\mathrm{i}$-th cluster, $i=1, \ldots, k$.

examples $\left(C_{i}\right)$ is the number of objects into the $\mathrm{i}$-th cluster, which are recognized by the concept $C_{i}$.

counterexamples $\left(C_{i}\right)$ is the number of objects outside of the i-th cluster, which are recognized by the concept $C_{i}$.

$\operatorname{Total}\left(C_{i}\right)$ is the number of objects in the i-th cluster.
} 
This function obtains higher values if the number of examples recognized by the concept increases and the number of counterexamples recognized by the concept decreases. The function obtains 1.0 when the concept recognizes all the objects in the cluster and it does not recognize any object outside the cluster, which is the ideal case.

For the example, the quality of the concepts obtained with each kind of support sets (see Table 4) is:

1) for the $\Gamma$-discriminating sets $=1.0$

2) for the $\Gamma$-characterizing sets $=1.0$

3) for the $\Gamma$-discriminating and $\Gamma$-characterizing sets $=1.0$

\section{Experimentation}

The CKMCF algorithm was applied on different supervised databases taken from the UCI repository [12].

For selecting the support sets, we did tests using different number of iterations: 10 , 20 and 30, and different number of individuals: 5, 10, 20, 50, 100 and 500. We obtained the best results with 10 iterations and 500 individuals. In Table 5, we show only the results obtained with the genetic algorithm with 10 iterations and 500 individuals, using the three kinds of support sets.

Table 5. Results obtained with the CKMCF algorithm using three kinds of support sets

\begin{tabular}{|c|c|c|c|c|c|c|c|}
\hline \multicolumn{8}{|c|}{ Conceptual K-means algorithm based on Complex Features } \\
\hline \multirow[b]{2}{*}{ Databases } & \multirow[b]{2}{*}{$\begin{array}{c}\text { No. } \\
\text { clusters }\end{array}$} & \multicolumn{2}{|c|}{$\Gamma d$} & \multicolumn{2}{|c|}{$\Gamma \mathrm{c}$} & \multicolumn{2}{|c|}{$\Gamma \mathrm{d}$ and $\Gamma \mathrm{c}$} \\
\hline & & $\begin{array}{c}\text { No. } \\
\text { predicates }\end{array}$ & Quality & $\begin{array}{c}\text { No. } \\
\text { predicates }\end{array}$ & Quality & $\begin{array}{c}\text { No. } \\
\text { predicates }\end{array}$ & Quality \\
\hline Autos_mpg & 3 & 29 & 0.69 & 29 & 0.69 & 29 & 0.69 \\
\hline Credit & 2 & 86 & 0.99 & 79 & 1.00 & 184 & 0.99 \\
\hline Diabetes & 2 & 63 & 0.72 & 62 & 0.72 & 64 & 0.72 \\
\hline Electro & 3 & 31 & 0.95 & 32 & 0.97 & 35 & 0.95 \\
\hline Glass & 6 & 23 & 0.99 & 23 & 0.95 & 23 & 0.98 \\
\hline Hayes & 3 & 32 & 1.00 & 31 & 1.00 & 32 & 1.00 \\
\hline Hepatitis & 2 & 69 & 0.75 & 58 & 0.95 & 59 & 0.87 \\
\hline Import85 & 6 & 97 & 0.65 & 60 & 0.87 & 104 & 0.94 \\
\hline Iris & 3 & 5 & 0.93 & 5 & 0.93 & 5 & 0.92 \\
\hline Lenses & 3 & 5 & 1.00 & 5 & 1.00 & 5 & 1.00 \\
\hline Tae & 3 & 24 & 0.98 & 24 & 0.98 & 24 & 0.98 \\
\hline Zoo & 7 & 28 & 1.00 & 19 & 1.00 & 27 & 1.00 \\
\hline Average & & 45.9 & 0.89 & 41.1 & 0.88 & 52.5 & 0.92 \\
\hline
\end{tabular}

In Table 5, we can observe that the results obtained with the different kinds of support sets, in most of the cases, are very similar. Therefore, we can use any of these support sets for obtaining good quality concepts. For the Hepatitis and the Import85 databases, we obtained lowest quality concepts using $\Gamma$-discriminating sets (see Figure 1). 


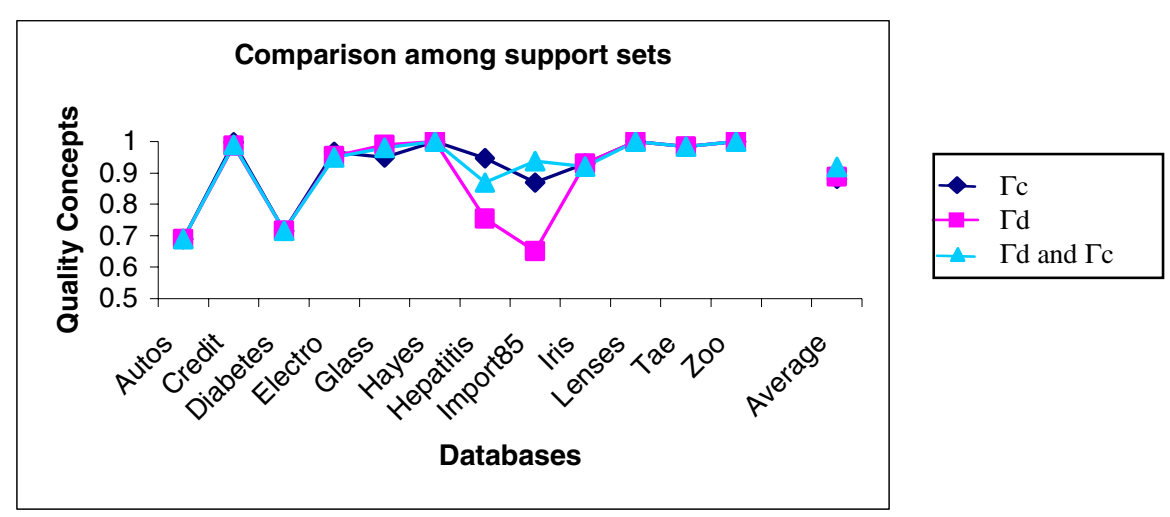

Fig. 1. Comparison among the three kinds of support sets for the CKMCF algorithm

\section{Comparison Between the CKM and the CKMCF Algorithms}

In this section, a comparison between the conceptual k-means (CKM) [8] and the conceptual k-means based on complex features (CKMCF) algorithms is presented. In Table 6, the results obtained by both algorithms are shown.

Table 6. Results obtained with the CKMCF and the CKM algorithms

\begin{tabular}{|c|c|c|c|c|c|c|c|c|c|}
\hline \multirow{3}{*}{ Databases } & \multirow{3}{*}{$\begin{array}{c}\text { No. } \\
\text { Clusters }\end{array}$} & \multicolumn{6}{|c|}{ CKMCF } & \multirow{2}{*}{\multicolumn{2}{|c|}{ CKM }} \\
\hline & & \multicolumn{2}{|c|}{$\Gamma d$} & \multicolumn{2}{|c|}{$\Gamma \mathrm{c}$} & \multicolumn{2}{|c|}{$\Gamma d$ and $\Gamma \mathrm{c}$} & & \\
\hline & & $\begin{array}{l}\text { No. } \\
\text { Pred }\end{array}$ & Quality & $\begin{array}{l}\text { No. } \\
\text { Pred }\end{array}$ & Quality & $\begin{array}{c}\text { No. } \\
\text { Pred }\end{array}$ & Quality & $\begin{array}{c}\text { No. } \\
\text { Pred }\end{array}$ & Quality \\
\hline Diabetes & 2 & 63 & 0.72 & 62 & 0.72 & 64 & 0.72 & 307 & 0.90 \\
\hline Glass & 6 & 23 & 0.99 & 23 & 0.95 & 23 & 0.98 & 39 & 0.84 \\
\hline Hayes & 3 & 32 & 1.00 & 31 & 1.00 & 32 & 1.00 & 26 & 0.97 \\
\hline Iris & 3 & 5 & 0.93 & 5 & 0.93 & 5 & 0.92 & 17 & 0.90 \\
\hline Lenses & 3 & 5 & 1.00 & 5 & 1.00 & 5 & 1.00 & 13 & 1.00 \\
\hline Tae & 3 & 24 & 0.98 & 24 & 0.98 & 24 & 0.98 & 13 & 0.96 \\
\hline Zoo & 7 & 28 & 1.00 & 19 & 1.00 & 27 & 1.00 & 13 & 0.99 \\
\hline Average & & 25.7 & 0.88 & 24.1 & 0.95 & 25.7 & 0.94 & 62.13 & 0.94 \\
\hline
\end{tabular}

Comparing the results obtained with the CKMCF and CKM algorithms, we can observe that, with the Diabetes database the CKM algorithm was better than the CKMCF algorithm. However, for the rest of databases the CKMCF algorithm obtains concepts with better quality than the concepts obtained by the CKM algorithm (Figure 2). In Table 6, we can see that, in average, the set of predicates obtained by the CKMCF algorithm is smaller than those obtained by the CKM algorithm, then the concepts obtained by the CKMCF algorithm are shorter and easier for handling than those obtained by the CKM algorithm.

We compared the CKMCF and the CKM algorithms using only six databases from those used in the previous section (see Table 5) since for the Autos_mpg, Credit, Electro, Hepatitis and Import85 databases was not possible to apply the CKM 
algorithm because these databases contain missing data, which cannot be processed by the CKM algorithm.

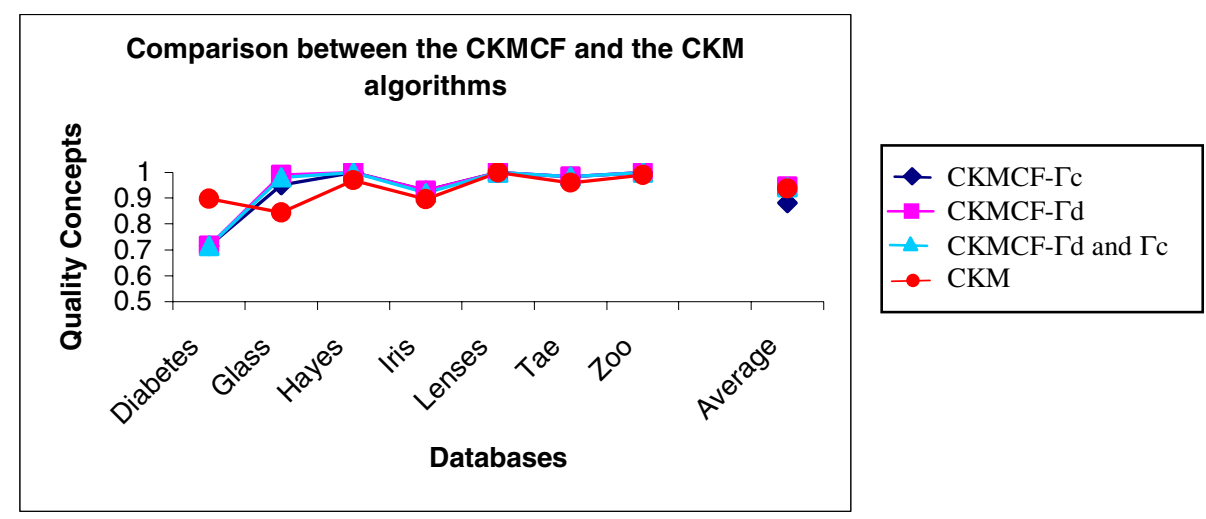

Fig. 2. Comparison between the CKMCF and the CKM algorithms

\section{Conclusions and Future Work}

In this paper, the conceptual k-means algorithm based on complex features (CKMCF) for solving conceptual clustering problems when the number of clusters is known a priori was proposed.

We consider that the CKMCF algorithm is a good alternative for the solution of conceptual clustering problems, because this algorithm allows working with mixed and missing data without any transformation of features; the centroids of the clusters are objects of the sample instead of elements that cannot be represented in the same space of the sample. Also, this algorithm does not depend on generalization lattices.

Based on the experimental results, we can observe that the CKMCF algorithm obtains very similar results with the three kinds of support sets. Then we can use any of these support sets for obtaining good quality concepts.

From the comparison between the CKMCF and the CKM algorithms we could observe that, in most of the cases, the CKMCF algorithm obtains better quality concepts with smaller set of predicates than those obtained by the CKM algorithm. Even though the number of predicates was decreased, the concepts of the clusters are formed by many predicates then as future work we will propose some modifications in order to generate smaller concepts.

\section{References}

1. Michalski R.S. (1986), A theory and methodology of inductive learning. In R.S. Michalski, J. G. Carbonell and T. M. Mitchell, editors, Machine Learning: An artificial intelligence approach, volume 2, pp. 83-129. Morgan Kaufmann, Los Altos, CA.

2. Briscoe G. and Caelli T. (1996), A compendium of Machine Learning, Volume 1: Symbolic Machine Learning, Ed. Ablex. 
3. Stumme G. et. al. (2001), Conceptual Clustering with Iceberg Concept Lattices, In: R. Klinkenberg, S. Ruping, A. Fick, N. Henze, C. Herzog, R. Molitor, O. Schroder (Eds.): Proc. GI-Fachgruppentre\#en Maschinelles Lernen '01,Universitat Dortmund 763.

4. Jonyer I., Holder L.B. and Cook D.J. (2001), Graph-based hierarchical conceptual clustering. International Journal on Artificial Intelligence Tools, 10 (1-2), pp. 107-135.

5. Pons-Porrata A., Ruiz-Shulcloper J. and Martínez-Trinidad J.F. (2002), RGC: a new conceptual clustering algorithm for mixed incomplete data sets. In Mathematical and Computer Modeling 36, pp. 1375-1385.

6. Osinski S. and Weiss D. (2004), Conceptual clustering using lingo algorithm: Evaluation on open directory project data. Advanced in Soft Computing, Intelligent Information Processing and Web Mining, Proceedings of the International IIS: IIPWM'04 Conference, Zakopane, Poland, pp. 369-378.

7. Jänichen S. and Perner P. (2005), Acquisition of Concept Descriptions by Conceptual Clustering, In P. Perner and A. Imiya (Eds.): MLDM 2005, LNAI 3587, pp. 153-162.

8. Ralambondrainy H. (1995), A conceptual version of the K-means algorithm, Pattern Recognition Letters 16, pp. 1147-1157.

9. De la Vega Doria, L.A, Carrasco Ochoa J.A. and Ruiz Shulcloper J. (1998), Fuzzy Kora- $\Omega$ Algorithm, 6th European Congress on Intelligent Techniques and Soft Computing EUFIT 98, Aachen Germany, vol. 2 pp. 1190-1194.

10. García Serrano J.R. and Martínez-Trinidad J.F. (1999), Extension to c-means algorithm for the use of similarity functions. 3rdEuropean Conference on Principles of Data Mining and Knowledge Discovery Proceedings. Prague, Czech. Republic, pp 354-359.

11. http://www.ics.uci.edu/pub/machine-learning-databases/ 